\title{
Scientometric Study of Product Lifecycle Management International Conferences: A Decade Overview.
}

\author{
Saurav BHATT ${ }^{1,3}$, Fen Hsuan TSENG ${ }^{2,3}$, Nicolas MARANZANA $^{3}$, Frédéric SEGONDS ${ }^{3}$ \\ ${ }^{1}$ Delhi College of Engineering, 110042 Delhi, India \\ ${ }^{2}$ National Taipei University of Technology, 10608 Taipei, Taiwan \\ ${ }^{3}$ Arts et Métiers ParisTech, LCPI, 151 boulevard de l'Hôpital, 75013 Paris, France \\ saurav_dce373@yahoo.co.in, karentseng211@gmail.com,nicolas.maranzana@ensam.eu, frederic.segonds@ensam.eu
}

\begin{abstract}
PLM International Conference proceedings focussing on the field of Product Lifecycle Management have made a lot of advancements in the last 12 years. Since 2003, 11 conferences on PLM have taken place but a systematic analysis of the evolution in PLM literature is, however, not available at the moment. This study proposes an analysis of the growth of the scientific literature on PLM over a 10 year period using standard bibliometric techniques. A total of 565 scientific papers have been examined to find out about the growth of literature, authorship pattern, geographical \& organizational distribution of papers, citation count and most frequently occurring keywords. The findings of this study give an insight into the evolution of literature on PLM by means of quantitative \& qualitative analysis and provide useful information to scientists wishing to undertake work in this field.
\end{abstract}

Keywords: Product Lifecycle Management, Bibliometrics, Citation Analysis, PLM Conference Proceedings

\section{Introduction and Literature Review}

PLM IC (Product Lifecycle Management International Conference) proceedings which are furthering research in the field of PLM have made a lot of advancements in the past 12 years. The first international conference on PLM took place in 2003 as an international symposium in India. The huge success of this symposium and growth of interest in the field of PLM led to running of further events as international conferences [1]. Since 2003, PLM conferences have been bringing together researchers, developers, and users of PLM involved in product innovation, product development, and product delivery together under one forum to share recent developments, shape the future of this field and advance the science and practice of enterprise systems development [2]. The conference series began to give PLM, as a research area, an identity and a community in the academic world. PLM holds the promise of seamlessly integrating and making available all of the information produced throughout all the stages of a product's lifecycle to everyone in an organization, along with key suppliers and customers [3]. The international conferences have been held 11 times and have published 565 papers since 2005 [4].

The present paper provides a bibliometric analysis of the PLM IC proceedings for the period 2005-2014. Bibliometrics is based on the enumeration and statistical analysis of scientific output in the form of articles, publications, citations, patents and other more complex indicators. It is an important tool to evaluate research activities, laboratories and scientists as well as the scientific specialisations and performance of countries [5]. The word 'bibliometrics' first appeared in 1969 in Alan Pitchard's article on statistical bibliography [6]. The term started getting widely used with the works of Lotka, Bradford and Zipf in the early $20^{\text {th }}$ century [7]. There are very few research papers that carried out the bibliometric analysis of PLM in the past. A. Varandas Junior et al. presented a bibliometric analysis of literature on 'Product Lifecycle Management, Product Development Process and Sustainability \& Their Interfaces [8]. The analysis was performed using various softwares and web of science database over a period of 5 years (2006-2010). Nappi and Rozenfeld also undertook a study on bibliometric analysis of research papers based on PLM in the paper entitled 'Sustainability Performance Indicators for Product Lifecycle Management' [9]. A research paper that investigates the contribution of PLM IC proceedings in the evolution of research work in PLM field is currently not available. The aim of this paper is to provide a review of the international conference proceedings on PLM over a 10 year period by means of bibliometric analysis.

The organisation of the research paper is done in a systematic way. Firstly, the methodology of extraction and treatment of data from the research papers to perform the analysis is described. Afterwards, outcomes of the bibliometric analysis of the research papers by qualitatively and quantitatively means are described and presented in the form of tables and figures. These outcomes help us perceive the evolution of the research in the field of PLM over a 10 year period. Lastly, the findings and conclusions of the paper are highlighted.

\section{Bibliometric Methodology}




\subsection{Introduction}

Bibliometrics is quantitative study of various aspects of literature on a topic and is used to identify the pattern of publication, authorship, and secondary journal coverage to gain insights into the dynamics of growth of knowledge in the areas under consideration [10]. The bibliometric study has been performed using the large database of PLM IC proceedings available on the www.plm-conference.org website. Though there are a lot of websites (Science Direct, Scopus, Google Scholar etc.) that provide huge database to perform bibliometric analysis of PLM, PLM IC proceedings website has been chosen to do the analysis as it is a platform that draws together people from all over the world pertaining to the field of PLM and provides large volume of data in a systemized and organised manner which is useful in doing fast analysis. In this study, bibliometric analysis of PLM for the period 2005-2014 has been performed. The analysis is performed from the year 2005 in which the International Conference proceeding on PLM took place in Lyon, France.

\subsection{Methodology for Citation Count \& Assignment of Contribution}

Furthermore, Google Scholar has been used to find out the keywords (whenever they were not available in the PLM IC database) and citation count of the papers as in contrast to websites like Web of Science and Scopus, Google Scholar provides a comprehensive database covering conference proceedings, journals, books, dissertations, and preprints [11]. The citation count of papers has been found out by using title and author search function of the Google Scholar. If the search engine doesn't list the searched research paper in the result, then the citation count of that paper is taken as zero. In sum, Google Scholar has been found out to be a very quick and efficient way to know the citation count of papers as it indexes a huge list of technical literature.

In this study, whenever a research paper is written by several countries or organizations in partnership, then the method of integer count rather than fractional count is adopted to assign the contribution of that particular country or organization. Integer count method has been preferred over fractional count method as it is in agreement with the principles of microanalysis. Also, integer count method is easier to implement and interpret [12]. Thus, in our study, for example, if both France and India published a paper in collaboration, then according to our methodology, France published one paper and India published one paper. In a similar way, if two authors (i.e. S. Charles \& G. Ducellier) published a paper together, then we say that S. Charles wrote one paper and G. Ducellier wrote one paper. Furthermore, if there are two offices of the same organizations or companies, then they are not considered as two separate entities but counted as one. Lastly, if two organizations (Arts et Métiers Paris Tech, France \& University of Michigan, America) published a paper together then we say that Arts et Métiers ParisTech, France published one paper and University of Michigan, America published one paper.

\subsection{Database}

In order to perform analysis of the large amount of information available on the PLM conference website, a selfdeveloped database was created using Microsoft Excel. The choice of Excel for making the database is based on the fact that it is one of the most famous powerful information management tool for organizing, calculating and visualizing data. The data extracted from all the 565 papers has been put into tabular form encompassing all the available information in 12 different columns, namely, year of publication, title of the paper, name of the authors, number of collaborating organizations, types of organizations, name of the organizations, number of collaborating countries, number of authors per paper, citation count of the paper, host country of the conference proceedings, number of keywords and names of the keywords. In addition to a general table encompassing all the available information, several other tables have been made to analyse the data and draw conclusions. Data corresponding to these tables have been obtained after some reasoning or further investigation while data for the general table has been retrieved directly from the research papers.

\section{Results of the Analysis: PLM International Conference Proceedings (2005-2014)}

The analysis of the data extracted from the research papers helps us find out about annual distribution of papers \& literature growth, geographical \& organizational distribution of papers, authorship patter \& degree of collaboration, the citation count of papers and the most frequently occurring keywords.

\subsection{Annual Distribution and Growth of Literature}


The organization of data obtained from the research papers has helped us visualize the growth of the scientific literature in the field of PLM over a 10 year period. There is a clearly a rise in the scientific literature involving PLM with total number of publications rising from 52 in the year 2005 to 565 in 2014 as shown in Fig. 1. The accumulated number of research papers published by PLM IC proceedings are shown in a year wise manner in Table 1. The row corresponding to total count for the number of countries, authors, and organizations for the period 2005-2014 considers counting the same author, country, and organization more than once as they may have participated more than once in the period 2005-2014.

Table 1. Most relevant data of the PLM IC proceedings for the period 2005-2014

\begin{tabular}{|c|c|c|c|c|c|c|}
\hline Year & Host City & Host Country & Papers & $\begin{array}{c}\text { No of } \\
\text { Countries }\end{array}$ & Authors & Organizations \\
\hline 2005 & Lyon & France & 52 & 18 & 146 & 63 \\
\hline 2006 & Bangalore & India & 29 & 9 & 80 & 40 \\
\hline 2007 & Stezzano & Italy & 85 & 25 & 260 & 104 \\
\hline 2008 & Seoul & Korea & 45 & 16 & 143 & 54 \\
\hline 2009 & Bath & UK & 68 & 20 & 227 & 86 \\
\hline 2010 & Bremen & Germany & 62 & 14 & 183 & 71 \\
\hline 2011 & Eindhoven & Netherlands & 46 & 15 & 128 & 56 \\
\hline 2012 & Montreal & Canada & 58 & 19 & 168 & 60 \\
\hline 2013 & Nantes & France & 70 & 23 & 211 & 81 \\
\hline 2014 & Tokyo & Japan & 50 & 18 & 157 & 70 \\
\hline Total & - & - & 565 & 177 & 1703 & 685 \\
\hline
\end{tabular}

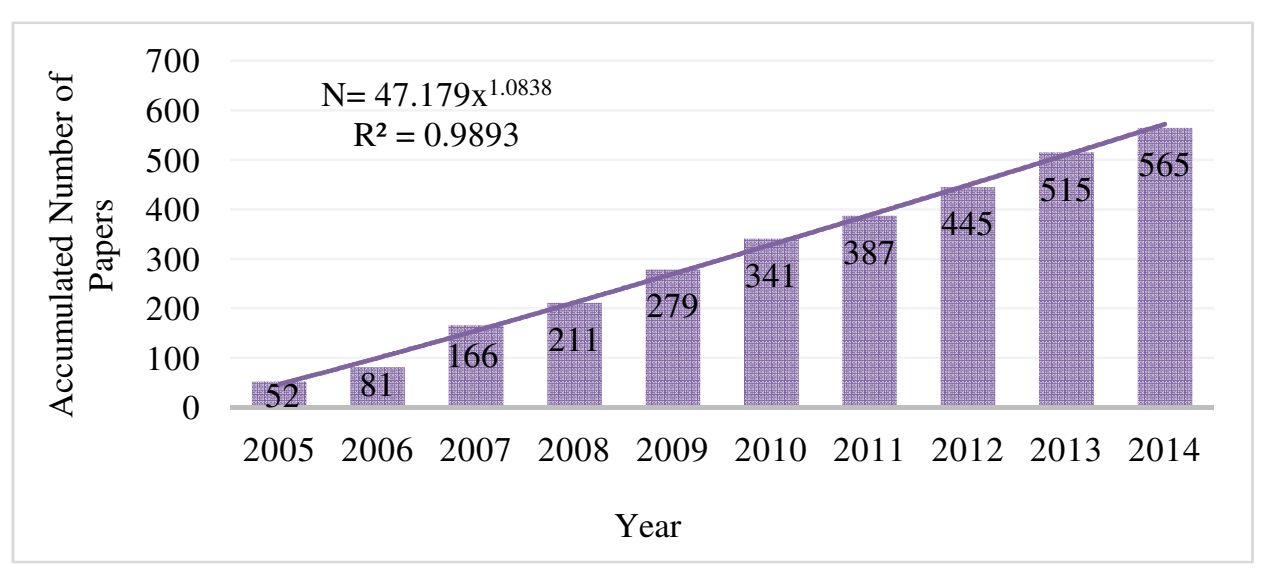

Fig. 1. Accumulated number of papers over a 10 Year Period. $\mathrm{N}$ being the accumulated number of papers and $\mathrm{x}$ stands for ordinal number of conferences.

The average number of papers published for the period 2005-2014 has been found to be 56.5 with the maximum number of papers published being 85 in the year 2007 with a participation of 25 countries, 260 authors and 104 organizations and the lowest number being 29 in the year 2006 with a participation of 9 countries, 80 authors and 40 organizations. Also, the calculated value of variance and standard deviation for the number of papers published over 10 years are 222.05 and 14.901. The fluctuations in the number of published papers might be because of a number of reasons like technological advances, degree of international collaboration, political policy etc.

\subsection{Geographical Distribution of Publications}

PLM IC proceedings have been attended by 41 countries during the period 2005-2014 with maximum number of contributions coming from France with a total number of 137 papers followed by Germany with 108 papers. Some of the other contributors are as follows: Italy (61 papers), UK (50 papers), USA (41 papers), South Korea (30 papers), Canada (28 papers), Finland (24 papers), India (22 papers), Japan (19 papers), Switzerland (18 papers), Sweden (16 papers), Netherlands (13 papers) and Australia (11 papers).

Therefore, France and Germany have been the major contributors at the PLM IC proceedings during the 10 year period and account for $43.4 \%$ of the total number of published papers. However, PLM based research work is 
getting a lot of attention and is distributed worldwide. The contribution of countries from all over the world is shown in Fig.2.

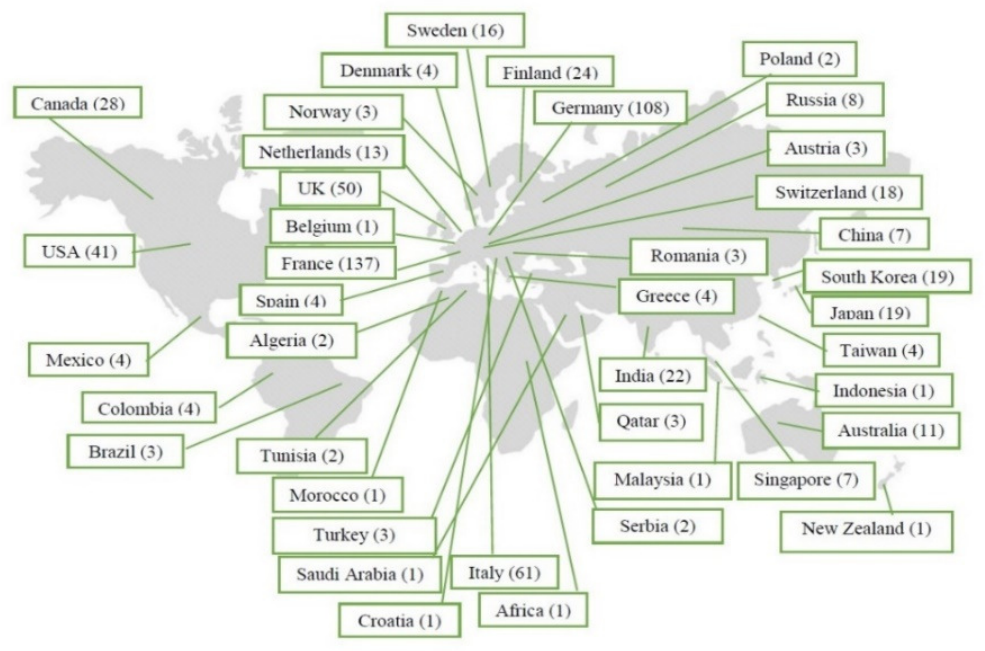

Fig. 2. Geographical distribution of the research papers

By noticing the distribution of international collaboration between different countries, it is found that the maximum number of collaborations has been found out to be between France \& UK with 6 publications, followed by France \& USA and France \& Canada with 5 publications, and lastly between France \& Italy and USA \& Japan with 4 publications.

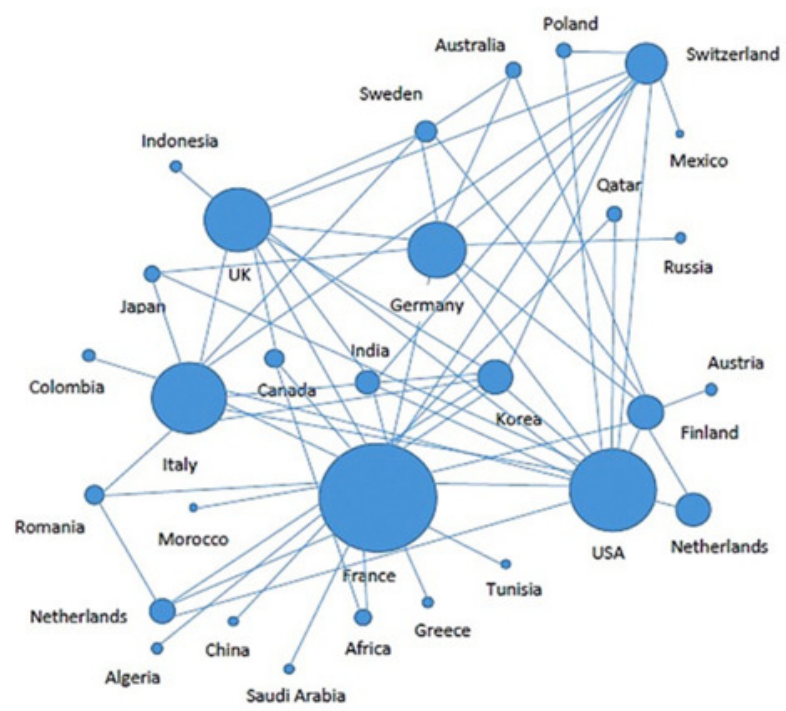

Fig. 3. Network diagram showing collaborations between different countries

Moreover, the country with the maximum number of international collaborations is France (19), followed by USA (12), Italy (11) and UK (10) and so on. The collaboration between different countries is shown using a network diagram in Fig. 3. In the network diagram, the size of the circles is proportional to number of international collaborations of that country. Thus, France with 19 international collaborations is depicted in the diagram using the biggest size circle while countries like Morocco, Columbia etc. having only 1 international collaboration have been shown using smallest size circle. It is easy to visualize from the network diagram that France, USA, UK, Italy, Germany, Switzerland are the countries with maximum number of international collaborations.

Lastly, majority of the collaborations are between two countries except for one between 7 countries (France, Italy, UK, USA, India, Korea, and Switzerland) in 2008, one between 4 countries (France, Italy, Netherlands, and Romania) in 2009, one between 3 countries (France, Finland, and Germany) in 2007, one between 3 countries (Poland, USA, and Switzerland) in 2009, one between 3 countries (France, Canada, and Africa) in 2010, and one between 3 countries (France, USA \& Qatar) in 2012 \& 2013. 


\subsection{Authorship Pattern and Degree of Collaboration}

Collaborative research instead of individualized one is a very important feature of the $21^{\text {st }}$ century. The distribution of authorship (Fig. 4.) clearly shows that out of 565 research papers, 521 (about 92\%) of the research papers have been written by multiple authors and only 44 research papers have been written by a single author. Table 2 . lists the number of papers written by single \& multiple authors per year. Clearly, each year the number of papers written by multiple authors far exceeds the number of papers written by single authors.

Table 2. Degree of Collaboration of authors

\begin{tabular}{|c|c|c|c|c|}
\hline Year & $\begin{array}{c}\text { No. of } \\
\text { Papers }\end{array}$ & $\begin{array}{c}\text { Single } \\
\text { Author }\end{array}$ & $\begin{array}{c}\text { Multiple } \\
\text { Authors }\end{array}$ & $\begin{array}{c}\text { Degree of } \\
\text { Collaboration }\end{array}$ \\
\hline 2005 & 52 & 10 & 40 & 0.80 \\
\hline 2006 & 29 & 3 & 26 & 0.90 \\
\hline 2007 & 85 & 9 & 76 & 0.89 \\
\hline 2008 & 45 & 1 & 44 & 0.98 \\
\hline 2009 & 68 & 2 & 66 & 0.97 \\
\hline 2010 & 62 & 3 & 59 & 0.95 \\
\hline 2011 & 46 & 5 & 41 & 0.89 \\
\hline 2012 & 58 & 2 & 56 & 0.97 \\
\hline 2013 & 70 & 6 & 64 & 0.91 \\
\hline 2014 & 50 & 3 & 47 & 0.94 \\
\hline Total & 565 & 44 & 521 & 0.92 \\
\hline
\end{tabular}

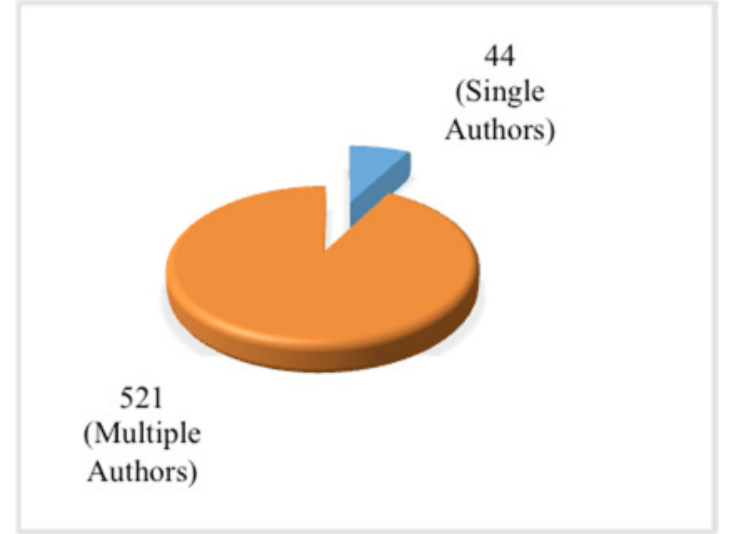

Fig. 4. Pie Diagram of Distribution of Authorship

The degree of collaboration calculated in Table 2 is found out using the mathematical formula (1) given by K. Subramanyam as follows:

$$
\text { Degree of Collaboration= NM/ (NM+NS). }
$$

where NM=No. of papers written by multiple authors and NS=No. of papers written by single authors. The overall degree of collaboration for a period of 10 years is found out to be 0.92 which clearly shows the importance of collaborative research. Furthermore, fig. 5. shows that the maximum number of the research papers (176) have been written by three authors in collaboration and is followed by four author (132) and two author case (129). As the number of co-authors become more than 5, the number of papers become very few in number (26).

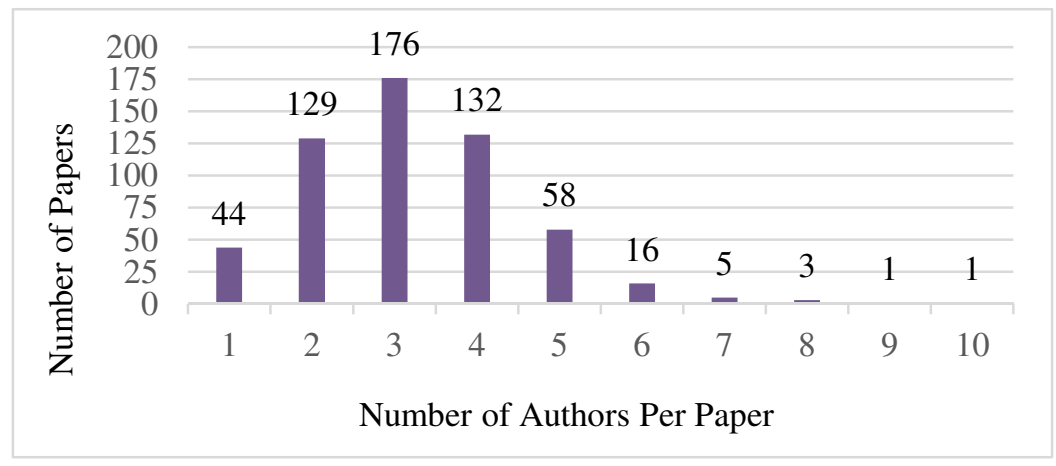

Fig. 5. Distribution of Co-Authorship

\subsection{Organizational distribution of papers}

As far as the organizations are concerned, University/Research Institutions (RI) turn out to be major contributors with $74.2 \%$ of the research papers (419 papers) coming from them. In contrast to this, only $9.4 \%$ of the research papers (53 papers) have been contributed by companies while contribution from company-university/research Institutions collaboration amount to $16.5 \%$ (93 papers). Fig. 6. shows that throughout the 10 year period universities and research institutions have contributed significantly in the growth of scientific literature in the field of PLM while the number is quite low for companies. 


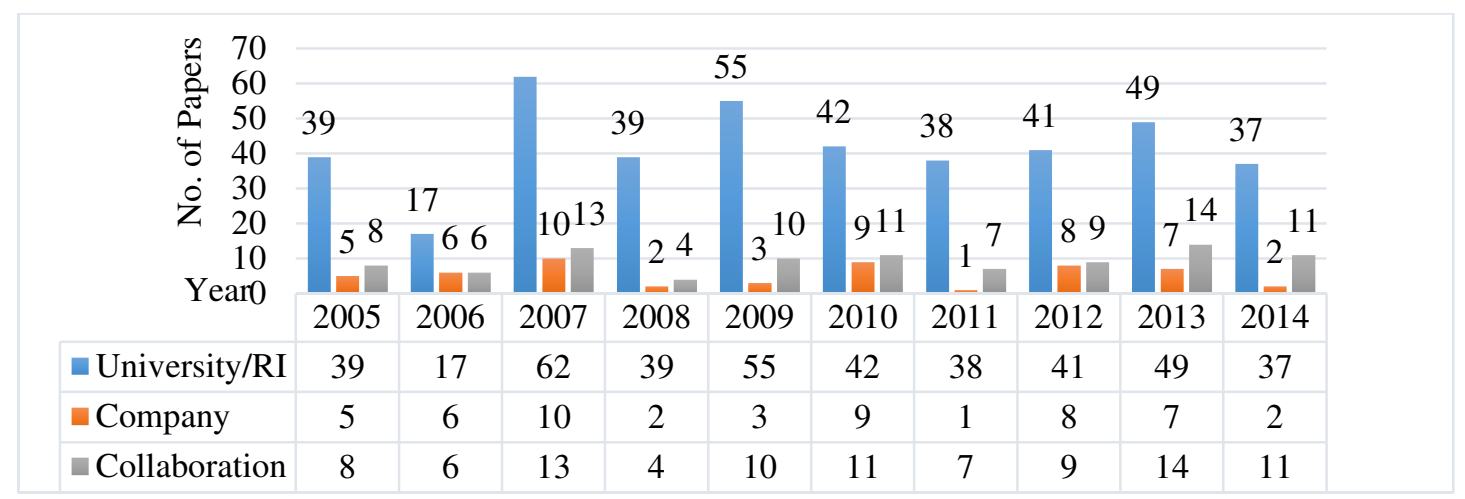

Fig. 6. Distribution of papers according to publishing organizations

Among the twenty of the most productive organizations, seven universities come from France alone. All the major contributors are universities with Université de Technologie de Compiègne \& Politecnico di Milano sharing the top place and contributing the maximum number of papers (21). The top 20 most productive organizations alone accounts for $48.9 \%$ (276 papers) of the total number of contributions.

\subsection{Citation Analysis}

Google Scholar has been chosen to perform the citations analysis of research papers because of its comprehensive database. The research papers have been searched in Google Scholar by using combination of title of the paper and author names to get an accurate count of number of citations. Furthermore, citation count for a research paper is taken as zero if the paper doesn't appear in the search results of Google Scholar. Table 3 lists the number of citations received by total number of papers (as on $26^{\text {th }}$ March, 2015) published each year with maximum number of citations being 300 for 52 research papers in the year 2005. In sum, a total of 1107 citations are obtained by 565 research papers over a 10 year period with average number of citations per research paper being 1.96 .

Table 3. Citation Pattern

\begin{tabular}{|c|c|c|c|}
\hline Year & No. of Articles & No of Citations & Average No. of Citations per Article \\
\hline 2005 & 52 & 300 & 5.769 \\
\hline 2006 & 29 & 37 & 1.276 \\
\hline 2007 & 85 & 142 & 1.670 \\
\hline 2008 & 45 & 96 & 2.133 \\
\hline 2009 & 68 & 209 & 3.074 \\
\hline 2010 & 62 & 75 & 1.210 \\
\hline 2011 & 46 & 84 & 1.826 \\
\hline 2012 & 58 & 95 & 1.638 \\
\hline 2013 & 70 & 59 & 0.843 \\
\hline 2014 & 50 & 10 & 0.200 \\
\hline Total & 565 & 1107 & 1.959 \\
\hline
\end{tabular}

\subsection{Keywords Classification}

Keywords mentioned in the research papers aid us in getting an insight into the focus of the research field of the papers. As there is no standard way in which keywords are mentioned, the authors vary the number of keywords used to outline the paper according to their own criterion while some of the authors don't mention it at all [13, 14]. Similar pattern has been observed while performing analysis of the 565 research papers of PLM IC proceedings. Some of the authors didn't mention the keywords at all [15] while others varied the number of keywords by large amount (the lowest number being 2 while the highest number going up to 23). The total number of keywords for all the 565 papers is calculated to be 2068 giving an average of 3.66 keywords per paper. 
Taking into consideration the diversity of the keywords found, the approach used in this research paper for classification of the keywords is to take into account only the first three keywords mentioned by the authors as they describe the contents of the research paper precisely. However, the shortcoming of this approach is that not all the research papers of the PLM IC proceedings have keywords.

Table 4. Top 10 most frequent keywords by taking into account first three author's keywords

\begin{tabular}{|c|c|c|c|}
\hline Number & Word & Rate of Occurrence & Weighted Percentage (\%) \\
\hline 1 & Product Lifecycle Management/PLM & 93 & 9.12 \\
\hline 2 & Modeling & 26 & 2.55 \\
\hline 3 & Knowledge Management & 18 & 1.76 \\
\hline 4 & Product Lifecycle & 12 & 1.18 \\
\hline 5 & Product Development & 9 & 0.88 \\
\hline 6 & Ontology & 9 & 0.88 \\
\hline 7 & PLM Implementation & 8 & 0.78 \\
\hline 8 & Production Management & 8 & 0.78 \\
\hline 9 & Production Engineering & 8 & 0.78 \\
\hline 10 & Interoperability & 8 & 0.78 \\
\hline
\end{tabular}

In order to calculate the rate of occurrence of keywords, NVivo 10 [16] software has been used. While performing word frequency query in NVivo, stop words such as conjunctions or prepositions are not taken into account as they are not meaningful in the analysis. Also, 'exact matching criteria' for the words is selected while performing word query analysis. If a keyword is made of more than one word, then the space between individual words of that keyword is omitted before running query otherwise NVivo breaks the keyword into separate words and gives rate of occurrence of individual words of that keyword. For example, the keyword 'Product Lifecycle Management' is written as 'ProductLifecycleManagement' before performing the query otherwise 'Product Lifecycle Management' is broken into 'Product', 'Lifecycle' and 'Management' by the software and the final result that is obtained is rate of occurrence of each of the individual words rather than that of 'Product Lifecycle Management' altogether. The principal outcomes given by the software are the most frequently occurring words, their citation count and their weighted percentage as shown in Table 4. As can be seen from the table, keyword 'Product Lifecycle Management' occupies the top place with a citation count of 53 followed by the keywords 'PLM', 'Modeling' and so on.

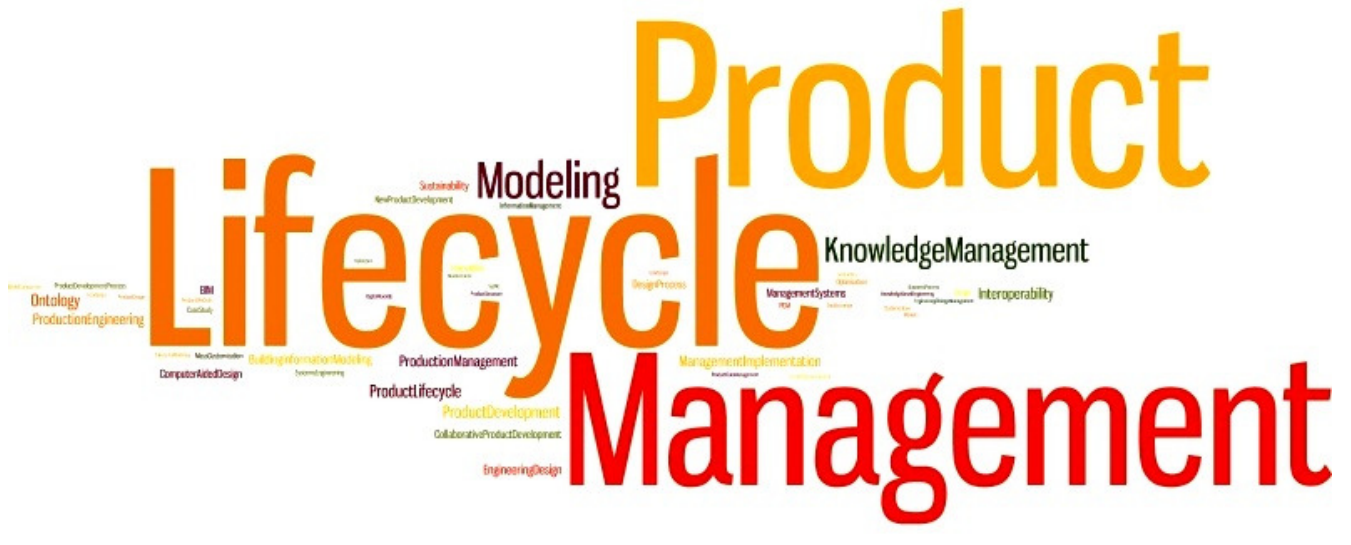

Fig. 7. Word Cloud made by using first three author's keywords

Apart from using NVivo, Wordle has been used to create word clouds using the first three of the author's keywords as the input text (Fig. 7.). The clouds give greater prominence to words that appear more frequently in the input text, thus helping us easily visualize the words appearing most frequently in the submitted text.

\section{Findings and Conclusions}


This study carried out a bibliometric analysis of 565 papers published by the PLM IC proceedings for the period 2005-2014. The analysis helped us find out the growth of literature over 10 years, the geographical \& organizational distribution of papers, authorship pattern, citation count of papers and the most frequently occurring keyword.

Though the number of articles published per year is not consistent and varies from year to year, there is clearly a rise in the scientific literature involving PLM with total number of publications rising from 52 in the year 2005 to 565 in 2014 . The average number of papers published for the period 2005-2014 has been found out to be 56.5. Geographical distribution of the papers shows that France and Germany are the major contributors at the PLM IC proceedings during the 10 year period and account for $43.36 \%$ of the total number of published papers. The maximum number of collaborations has been found out to be between France \& UK with 6 publications and the country with the maximum number of international collaborations is France with 19 tie-ups in total. Furthermore, 521 (about 92\%) research papers have been written by multiple authors and only 44 research papers have been written by single author. The most dominant is found out to be the 3 authors' case. Hence, collaborative research is prominent in this field with an average degree of collaboration of 0.922 . Throughout the 10 year period, universities and research institutions have contributed significantly in the growth of scientific literature in the field of PLM while the number is quite low for companies. Universities/Research institutions account for $74.16 \%$ (419) of the total research papers while only 9.38\% (53) of the research papers are contributed by companies. Citation analysis shows that a total of 1107 citations have been obtained by 565 research papers over a 10 year period leading to an average number of citations per research paper of 1.959 . Lastly, there is a lot of diversity in the number of keywords provided by the authors. Some of the authors didn't mention the keywords at all while others varied the number of keywords by a large amount (the lowest number being 2 while the highest number going up to 23). The total number of keywords for all the 565 papers is calculated to be 2068 giving an average of 3.66 keywords per paper.

The findings of this study provide us with useful information about the state of PLM based research in the world. PLM is an evolving discipline that is attracting attention from all over the world. Results of the study depend on the chosen data source. The methodology mentioned in this paper has been applied to database of PLM IC proceedings for a period of 10 years only to arrive at meaningful results in order to get an insight into the evolution of research work done in the field of PLM. It would be worth applying this methodology to other databases and time periods to arrive at comparative results. For the time being, we can say that this study gives a broad view of PLM IC proceedings effort to promote research work in the field of PLM by bibliometric analysis and will prove very useful to perform similar bibliometric studies in the field of PLM in the near future.

\section{References}

$[1,3]$ http://www.inderscience.com/browse/book.php?journalID=1001\&action=editorial

$[2,4]$ http://www.plm-conference.org/index.php (visited on $20^{\text {th }}$ March, 2015)

[5] Okubo, Y., "Bibliometric Indicators and Analysis of Research Systems: Methods and Examples", OECD Science, Technology and Industry Working Papers, 1997/01, OECD Publishing (1997)

[6] Pritchard, Alan., "Statistical Bibliography or Bibliometrics", Journal of Documentation, 25(4): pp. 348-349 (1969)

[7] Thanuskodi, S., "Bibliometric analysis of Indian Journal of Agricultural Research", International Journal of Information Dissemination and Technology, 2(3), pp. 170-175 (2012)

[8] Varandas, A., Augustol, P., Carvalho, M., "Product Lifecycle Management, Product Development Process and Sustainability \& Their Interfaces", $3^{\text {rd }}$ International Workshop on Advances in Cleaner Production, Sao Paulo, Brazil (2015) [9] Nappi, V. and Rozenfeld, H., "Sustainability Performance Indicators for Product Lifecycle Management", 22 ${ }^{\text {nd }}$ International Congress of Mechanical Engineering (COBEM 2013), Sao Paulo, Brazil (2013)

[10] Thanuskodi, S., "Bibliometric Analysis of the Journal Library Philosophy and Practice from 2005-2009", Library Philosophy and Practice, pp. 437 (2010)

[11] Yang, K. and Meho, L.I., "Citation Analysis: A Comparison of Google Scholar, Scopus, and Web of Science", $69^{\text {th }}$ Annual Meeting of the American Society for Information Science and Technology (ASIST), Austin, Texas, USA (2006)

[12] The Observatory of Science and Technology, "Bibliometrics as a tool for the analysis of the scientific production of a country", Paris, France (2009)

[13] Hervy, B., Laroche, F., Bernard, A., Kerouanton, J., "Co-working for Knowledge Management in Cultural Heritage: Towards a PLM for Museum", Product Lifecycle Management for Society, IFIP Advances in Information and Communication Technology, Volume 409, pp. 317-325 (2013)

[14] Pintzos, G., Rentzos, L., "A Knowledge Based Collaborative Platform for the Design \& Deployment of Manufacturing Systems", IFIP Advances in Information \& Communication Technology, Volume 409, pp. 268-276 (2013)

[15] Barbau, R., Lubell, J., Rachuri, S., Foufou, S., "Toward a Reference Architecture for Archival Systems", IFIP Advances in Information and Communication Technology, Volume 409, pp. 68-77 (2013)

[16] http://www.qsrinternational.com/products_nvivo.aspx (visited on 27 $7^{\text {th }}$ March, 2015) 